\title{
Fluorescence in situ hybridization status of voided urine predicts invasive and high-grade upper tract urothelial carcinoma
}

\author{
Xiaohong Su${ }^{1,2, *}$, Han Hao ${ }^{1,2, *}$, Xuesong $\mathrm{Li}^{1,2}$, Zhisong $\mathrm{He}^{1,2}$, Kan Gong ${ }^{1,2}$, Cuijian \\ Zhang ${ }^{1,2}$, Lin $\mathrm{Cai}^{1,2}$, Qian Zhang ${ }^{1,2}$, Lin $\mathrm{Yao}^{1,2}$, Yi Ding ${ }^{1,2}$, Yanqing Gong ${ }^{1,2}$, Dong \\ Fang $^{1,2}$, Zheng Zhang ${ }^{1,2}$, Liqun Zhou ${ }^{1,2}$ \\ ${ }^{1}$ Department of Urology, Peking University First Hospital, Xicheng, Beijing, China \\ ${ }^{2}$ Institute of Urology, Peking University, National Urological Cancer Center, Beijing, China \\ "These authors contributed equally to this work \\ Correspondence to: Liqun Zhou, email: zhoulqmail@sina.com \\ Zheng Zhang, email: doczhz@aliyun.com \\ Keywords: fluorescence in situ hybridization (FISH), upper tract urothelial carcinoma (UTUC), high grade, radical nephroure- \\ terectomy $(R N U)$, conservative management \\ Received: September 23, $2016 \quad$ Accepted: January 24, $2017 \quad$ Published: February 15, 2017 \\ Copyright: Su et al. This is an open-access article distributed under the terms of the Creative Commons Attribution License (CC-BY), which \\ permits unrestricted use, distribution, and reproduction in any medium, provided the original author and source are credited.
}

\section{ABSTRACT \\ Here, we assessed the diagnostic accuracy of fluorescence in situ hybridization (FISH) for detecting aggressive upper tract urothelial carcinoma (UTUC). A total of 210 UTUC patients from a single hospital in China were enrolled in this retrospective study between 2012 and 2016. Patients were classified as FISH-positive or -negative based on FISH analysis of voided urine, and the clinicpathological characteristics of these two patient groups were compared. Patients with positive FISH results had more advanced tumor stages and higher tumor grades than those with negative results. The overall sensitivity of FISH for detecting advanced UTUC was $62.4 \%(131 / 210)$. The sensitivity and positive predictive values of positive FISH results were $76.5 \%$ and $59.5 \%$ for high-grade UTUC and $\mathbf{7 1 . 7 \%}$ and $\mathbf{5 8 . 0 \%}$ for muscle-invasive UTUC. These data suggest that voided urine FISH results accurately predict advanced UTUC and might help distinguish patients with aggressive disease from those who might benefit from conservative management.}

\section{INTRODUCTION}

Upper tract urothelial carcinomas (UTUCs) are relatively rare compared to bladder cancer and account for approximately $5 \%$ to $10 \%$ of urothelial carcinomas [1]. Radical nephroureterectomy (RNU) with an ipsilateral bladder cuff excision, which is the gold-standard treatment for UTUC [2], can result in varying degrees of renal insufficiency. Therefore, conservative management of small, low-grade and non-invasive UTUC may help preserve renal function in select groups of patients [2], and accurate pre-oprative risk stratification of UTUC patients is crucial. However, accurately determining the stage and grade of UTUC via urine cytology or preoperative imaging is difficult. In addition, neither ureteroscopy nor urine cytology is sufficient for accurately predicting muscle invasion or the risk of high-grade disease in UTUC patients $[3,4]$. Thus, novel, more reliable methods are needed to identify low-risk patients before surgery.

Fluorescence in situ hybridization (FISH) analysis of voided urine has become a popular aid for initial diagnosis and subsequent monitoring for recurrence in bladder cancer patients, and previous studies have confirmed that it is highly sensitive and specific $[5,6]$. Here, we investigated the ability of FISH to predict high grade and muscle-invasive tumors, and thus to distinguish tumors for which conservative approaches to treatment would be appropriate from those that should be treated with RNU.

\section{RESULTS}

The clinical and pathological characteristics of the patients are listed in Table 1 and are stratified according to the FISH results. There were 102 male and 108 female patients with a median age of 70.5 years (inter-quartile range [IQR]: 63-75 years). The diagnosis of urothelial carcinoma was pathologically confirmed in all patients; 104 had non-muscle-invasive diseases ( $\leq$ pT1), and 106 had muscle-invasive diseases $(\geq \mathrm{pT} 2)$, while 108 had low grade and 102 had high grade diseases. Conservative surgeries were performed in 21 patients; 7 of the patients had non-muscle-invasive $(\leq \mathrm{pT} 1)$ and 14 had muscle- 
Table 1: Clinical and pathological characteristics of UTUC patients according to FISH status

\begin{tabular}{|c|c|c|c|c|c|}
\hline & & \multicolumn{2}{|c|}{ FISH } & \multirow{2}{*}{ Chi-square or $\mathrm{Z}$} & \multirow{2}{*}{$p$ value } \\
\hline & & Positive & Negative & & \\
\hline \multicolumn{2}{|l|}{ Median age } & $68.81 \pm 10.35$ & $67.10 \pm 9.52$ & -1.460 & 0.144 \\
\hline \multirow[t]{2}{*}{ Gender } & Male & 70 & 32 & 3.298 & 0.069 \\
\hline & Female & 61 & 47 & & \\
\hline \multirow[t]{2}{*}{ Hydronephrosis } & Present & 20 & 15 & 0.491 & 0.483 \\
\hline & Absent & 111 & 64 & & \\
\hline \multirow[t]{3}{*}{ Tumor location } & Pelvis & 63 & 29 & 3.059 & 0.217 \\
\hline & Ureter & 60 & 46 & & \\
\hline & Both & 8 & 4 & & \\
\hline \multirow[t]{5}{*}{ Tumor stage } & A & 6 & 11 & 13.709 & 0.008 \\
\hline & 1 & 49 & 38 & & \\
\hline & 2 & 32 & 18 & & \\
\hline & 3 & 43 & 11 & & \\
\hline & 4 & 1 & 1 & & \\
\hline \multirow[t]{2}{*}{ Tumor grade } & High & 78 & 24 & 16.778 & $<0.001$ \\
\hline & Low & 53 & 55 & & \\
\hline \multirow[t]{2}{*}{ Lymph node status } & $\mathrm{cN} 0$ or $\mathrm{pN} 0$ & 123 & 77 & 1.389 & 0.239 \\
\hline & $\mathrm{N}+$ & 8 & 2 & & \\
\hline \multirow[t]{2}{*}{ LVI } & Presence & 20 & 9 & 0.622 & 0.430 \\
\hline & Absence & 111 & 70 & & \\
\hline \multirow[t]{2}{*}{ Tumor size } & $\leq 3 \mathrm{~cm}$ & 72 & 45 & 0.080 & 0.777 \\
\hline & $>3 \mathrm{~cm}$ & 59 & 34 & & \\
\hline \multirow[t]{2}{*}{ Multifocal } & Yes & 24 & 10 & 1.164 & 0.281 \\
\hline & No & 107 & 69 & & \\
\hline
\end{tabular}

FISH = fluorescence $i n$ situ hybridization; LVI = lymphovascular invasion.

Table 2: Preoperative factors that predict high-risk UTUC in univariate and multivariate analyses

\begin{tabular}{|c|c|c|c|c|c|c|c|c|c|c|c|}
\hline \multirow{2}{*}{ Preoperative factors } & & \multicolumn{2}{|c|}{ Tumor Stage } & \multirow{2}{*}{$\begin{array}{c}\text { Univariate } \\
p\end{array}$} & \multicolumn{2}{|l|}{ Multivariate } & \multicolumn{2}{|c|}{ Tumor Grade } & \multirow{2}{*}{$\frac{\text { Univariate }}{p}$} & \multicolumn{2}{|c|}{ Multivariate } \\
\hline & & Ta-1 & $\geq \mathrm{pT} 2$ & & HR $(95 \%$ CI) & $p$ & Low grade & High grade & & HR $(95 \%$ CI) & $p$ \\
\hline Patients & & 104 & 106 & & & & 108 & 102 & & & \\
\hline \multirow[t]{2}{*}{ Gender } & Male & 45 & 57 & 0.128 & $1.233(0.695-2.189)$ & 0.474 & 48 & 54 & 0.218 & $1.244(0.705-2.194)$ & 0.451 \\
\hline & Female & 59 & 49 & & & & 60 & 48 & & & \\
\hline \multirow[t]{2}{*}{ Age } & $\leq 70$ & 54 & 51 & 0.581 & $1.166(0.662-2.055)$ & 0.594 & 57 & 48 & 0.407 & $1.234(0.696-2.189)$ & 0.472 \\
\hline & $>70$ & 50 & 55 & & & & 51 & 54 & & & \\
\hline \multirow[t]{2}{*}{ FISH } & Positive & 55 & 76 & 0.005 & $2.266(1.272-4.036)$ & 0.005 & 53 & 78 & $<0.001$ & $3.373(1.864-6.102)$ & $<0.001$ \\
\hline & Negative & 49 & 30 & & & & 55 & 24 & & & \\
\hline \multirow[t]{3}{*}{ Hydronephrosis } & Present & 14 & 21 & 0.217 & $1.674(0.782-3.586)$ & 0.185 & 19 & 16 & 0.711 & $0.936(0.425-2.058)$ & 0.869 \\
\hline & Absent & 90 & 85 & & & & 89 & 86 & & & \\
\hline & Pelvis & 50 & 42 & 0.446 & $0.770(0.471-1.261)$ & 0.300 & 45 & 47 & 0.563 & $1.177(0.733-1.891)$ & 0.500 \\
\hline \multirow[t]{2}{*}{ Tumor location } & Ureter & 49 & 57 & & & & 58 & 48 & & & \\
\hline & Both & 5 & 7 & & & & 5 & 7 & & & \\
\hline \multirow{2}{*}{ Tumor size } & $\leq 3 \mathrm{~cm}$ & 65 & 52 & 0.05 & $1.741(0.993-3.050)$ & 0.053 & 62 & 55 & 0.611 & $1.083(0.601-1.953)$ & 0.791 \\
\hline & $>3 \mathrm{~cm}$ & 39 & 54 & & & & 46 & 47 & & & \\
\hline \multirow[t]{2}{*}{ Multifocal } & Yes & 16 & 18 & 0.754 & $0.985(0.443-2.190)$ & 0.971 & 16 & 18 & 0.578 & $1.014(0.454-2.262)$ & 0.973 \\
\hline & No & 88 & 88 & & & & 92 & 84 & & & \\
\hline
\end{tabular}

$\mathrm{HR}=$ harzard ratio; $\mathrm{CI}=$ confidence interval; FISH $=$ fluorescence in sit $u$ hybridization.

invasive ( $\geq$ pT2) UTUC, and 12 had low grade and 9 had high grade diseases.

FISH analysis of voided urine from 131 patients returned "positive" results, indicating the presence of chromosomal abnormalities, while FISH results for the remaining 79 patients were negative. No significant differences in age, gender, hydronephrosis, tumor location, lymph node status, lymphovascular invasion (LVI), tumor size, or tumor focality were observed between the positive and negative FISH groups (all $p>0.05$ ). However, patients with positive FISH results had more advanced tumor stages $(p=0.008)$ and higher tumor grades than negative patients $(p<0.001)$. In binary logistic regression, after adjusting for clinical and pathological factors, only preoperative positive FISH status was an independent risk factor for high-grade or muscle-invasive UTUC (Table 2). 


\section{Table 3: Sensitivity and positive predictive value of positive FISH status for predicting high-grade and muscle-invasive UTUC}

Voided urine FISH $(n=210)$

\begin{tabular}{ll}
\hline High-grade cancer & \\
$\quad$ Sensitivity & $76.47(67.04-84.31)$ \\
$\quad$ Positive predictive value & $59.54(50.62-68.02)$ \\
Muscle-invasive cancer $(\geq$ pT2) & \\
Sensitivity & $71.70(62.12-80.02)$ \\
Positive predictive value & $58.02(49.08-66.58)$ \\
\hline
\end{tabular}

FISH afforded an overall sensitivity of $62.4 \%$ $(131 / 210)$ in all of the UTUC patients; the FISH status of patients after stratification by both pathological stage and grade is shown in Table 3. Positive FISH results had a sensitivity and PPV of $76.5 \% \%$ and $59.5 \%$ for highgrade UTUC and of $71.7 \%$ and $58.0 \%$ for muscle-invasive UTUC, respectively. Among the 79 patients with negative preoperative $\mathrm{FISH}$ results, 30.4\% had high-grade and $36.7 \%$ had muscle-invasive UTUC upon final pathology.

\section{DISCUSSION}

Conservative management of low-risk UTUCs (which are characterized by unifocal tumors, tumor sizes $<1 \mathrm{~cm}$, low grade tumors, and no evidence of infiltrative lesions on CT urography) allows the renal unit to be preserved and spares the patient from morbidity associated with radical surgery [2]. The size of a tumor and whether it is unifocal or multifocal can be easily determined via preoperative imaging. However, it is difficult to accurately determine the stage and grade of UTUC using currently available methods. For example, Messer et al. demonstrated in a multi-institutional study of 326 patients that urine cytology alone was also unable to accurately predict muscle-invasive or high-grade disease in UTUC patients; positive urinary cytology had a sensitivity and PPV of only $56 \%$ and $54 \%$ for high-grade UTUC, and $62 \%$ and $44 \%$ for muscle-invasive UTUC, although restricting analysis to patients with selective ureteral cytology improved the diagnostic accuracy [4]. Although ureteroscopy is more sensitive and specific in diagnosing UTUC than urine cytology and radiography [7], it may not be accurate for predicting tumor grade and stage. In an early study, Keeley et al. demonstrated that ureteroscopy accurately predicted true tumor grade and stage as indicated by open surgical specimens of upper tract transitional cell carcinoma [8]. However, Straub et al. [3] found that the accuracy of preoperative ureteroscopy in predicting the correct tumor grade in UTUC patients was only $58 \%$; this accuracy only increased to $68 \%$ when urinary cytology was considered as well. Furthermore, ureteroscopy is an invasive procedure that requires general anesthesia and involves the accompanying surgical complications, and recent studies have identified diagnostic ureteroscopy as an independent risk factor for intravesical recurrence [9-12].
Diagnostic tools, or combinations of tools, that are effective in determining UTUC stage and grade are urgently needed to identify patients for whom conservative management is the most appropriate treatment strategy. Because urothelial carcinoma affects the entire urothelium, the lower and upper urinary tracts share many histological and cytological characteristics in this disease [13]. It is therefore plausible that FISH, which is commonly used in bladder cancer, would be an effective noninvasive, adjunct technique for detecting UTUC. To date, few studies have focused on the utility of FISH for accurately predicting tumor stage and grade in UTUC patients. Johannes et al. found that the sensitivity of FISH status of voided urine for predicting low-grade $(60 \%)$ and high-grade $(50 \%)$ UTUC was relatively low in a retrospective study with a small patient population [14]. However, Chen et al. retrospectively conduced FISH analysis on 94 specimens taken from 43 patients who were monitored for UTUC and found that its sensitivity was higher for predicting highgrade tumors (79\%) than for low-grade tumors (41\%) [15].

Here, $62.4 \%$ of UTUC patients had positive FISH results, which is consistent with previous studies in which the overall sensitivity of voided FISH for detecting or monitoring UTUC varies from $35 \%$ to $87.5 \%$ [15-19]. Moreover, our data suggest that positive FISH status, which had sensitivities and PPVs of 76.5\% and 59.5\% for highgrade UTUC and $71.7 \%$ and $58.0 \%$ for muscle-invasive UTUC, respectively, was the only independent risk factor for advanced UTUC. Due to its relatively high accuracy in predicting these advanced characteristics, voided urine FISH analysis may be helpful in distinguishing tumors best treated using conservative management from those that should be treated with RNU. Radical surgery would be recommended for patients with positive FISH results, whereas conservative surgeries, such as ureteroureterostomy or complete distal ureterectomy and neocystostomy, would be recommended for those with negative FISH results, especially for those with renal insufficiency or a solitary kidney.

The EAU guidelines for UTUC emphasize the importance of tumor multifocality when making decisions regarding the use of RNU or conservative management during treatment. In Chinese populations, the use of traditional Chinese medicine containing aristolochic acid (AA) might affect tumor multifocality, and a previous study of UTUC in Balkan endemic nephropathy patients 
indicated that malignancy was lower in UTUCs caused by AA [20]. Our previous research also suggests specific treatments that may be most effective for multiple or bilateral UTUC [21, 22], but choosing the best surgical approach remains difficult. By accurately predicting tumor stage and grade, FISH might help improve treatment decisions for these UTUC patients.

Some limitations of our study should be considered when interpreting the results. First, selection bias may have influenced the results of this nonrandomized, retrospective study. In addition, the radical and conservative surgical approaches selected by the surgeons and/or the patients were not based on preoperative FISH results. Finally, we were not able to evaluate the relationship between FISH status and oncologic prognosis due to the short followup period. Despite these limitations, this is the largest single-center study to date of the ability of FISH to predict UTUC stage and grade, and is the first report involving Chinese patients. Although a large-volume prospective study should be conducted to confirm these findings, our results suggest that preoperative FISH analysis of voided urine, which accurately predicted UTUC tumor stage and grade, may be a useful tool in guiding treatment decisions for UTUC patients.

\section{MATERIALS AND METHODS}

\section{Patient selection}

A total of 232 patients who underwent surgeries for UTUC in the Urology Department, Peking University First Hospital between January 2012 and May 2016 were enrolled in this study. Twenty-two patients with previous or concomitant bladder tumors were subsequently removed from the analysis. Clinicopathologic data for the remaining 210 patients were retrospectively reviewed. None of the patients had received neoadjuvant chemotherapy. The ethics committee of Peking University First Hospital approved the study.

\section{Treatment and evaluation}

One hundred and eighty-nine patients underwent RNU with an ipsilateral bladder cuff excision; the remaining 21 patients underwent conservative surgery, including ureteroureterostomy and complete distal ureterectomy and neocystostomy.

All pathological specimens were reexamined by a dedicated genitourinary pathologist to confirm the original diagnosis. Tumor stage was assessed according to the Union Internationale Contre le Cancer (UICC) 2009 Tumor Node Metastasis (TNM) classification of malignant tumors. Tumor grade was assigned according to the 2004 World Health Organization classification grading system. Tumor location was classified as ureter, renal pelvis, or both. Tumor multifocality was defined as the simultaneous presence of two or more pathologically confirmed macroscopic tumors in any location. Tumor size was defined as the maximal diameter of the lesion. The status of regional lymph nodes was based on the final pathological specimens. For patients with advanced tumor stages, lymph node metastasis, or postoperative local recurrence, adjuvant radiotherapy or chemotherapy were sometimes administered.

\section{Criteria for positive FISH}

Voided urine specimens from the 210 UTUC patients were analyzed using FISH; labeled probes specific for chromosomes 3, 7, and 17, and the p16 (9p21) genes were used to assess chromosomal abnormalities indicative of malignancy.

Briefly, FISH signals were examined in $>25$ morphologically abnormal, potentially malignant cells per patient. Abnormal signals (Figure 1) were defined as
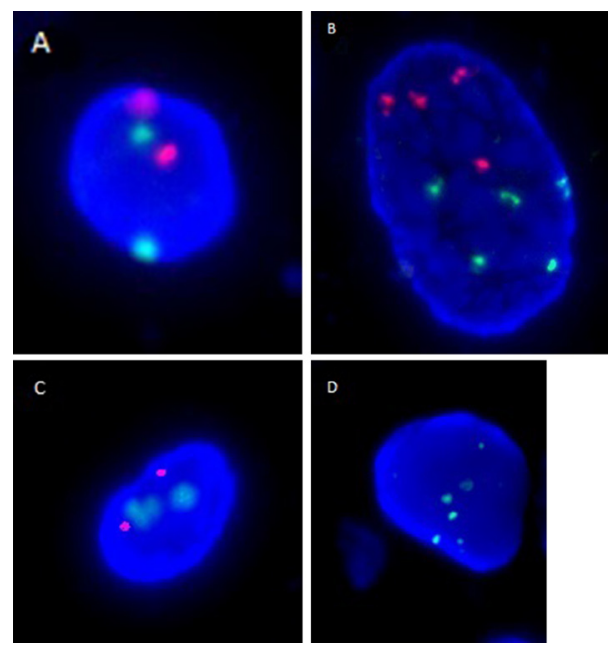

Figure 1: Representative FISH images are shown for (A) a normal urothelial cell with diploidy of chromosomes 3 (red signal) and 7 (green signal), (B) a cell with polyploidy of chromosomes 3 and 7, (C) a normal urothelial cell with diploidy of p16 genes (red signal) and chromosome 17 (green signal), and (D) a cell with polyploidy of chromosome 17 and homozygous deletion of p16 genes. 
either detection of three or more signals for chromosomes 3,7 , and 17 or the presence of heterozygous (-p16(-1)) or homozygous (-p16(-2)) deletions of p16 gene in the counted cells. A sample was considered FISH-positive for urothelial cancer if at least 4 cells that had two or more abnormal chromosomes (chromosomes 3, 7, 17 or -p16(-1)) were identified and/or if at least 12 cells with $-\mathrm{p} 16(-2)$ were observed.

\section{Statistical analysis}

Independent-sample Mann-Whitney $U$ and chisquared tests were used to compare continuous and categorical variables, respectively. Binary logistic regression was used to evaluate preoperative factors. Sensitivity and positive predictive values were calculated using FISH as the diagnostic test and tumor grade (high vs. low) or stage (muscle-invasive vs. non-muscleinvasive) as oncologic outcomes. All statistical analyses were conducted using SPSS version 20.0 (IBM Corp, Armonk, NY). All reported $p$-values are two sided; statistical significance was set at $<0.05$.

\section{Authors' contributions}

Xiaohong $\mathrm{Su}$ and Han Hao are co-first authors and contributed equally to the drafting and revising the manuscript and to the study.

Liqun Zhou and Zheng Zhang are corresponding authors and contributed to the overall study design. Xuesong Li, Zhisong He, Kan Gong, Cuijian Zhang, Lin Cai, Qian Zhang, Lin Yao, Yi Ding, Yanqing Gong, and Dong Fang contributed to data collection and analysis.

\section{ACKNOWLEDGMENTS}

This work was supported by grants from the Peking University Clinical Research Project (PUCRP201302), the Collaborative Research Foundation of Peking University Health Science Center and National Taiwan University, the College of Medicine (BMU20120318), the National Natural Science Foundation of China (81172419, 81372746), the Natural Science Foundation of Beijing (7122183, 7152146), the Clinical Features Research of the Capital (No. Z151100004015173) and the Research Foundation of Peking University First Hospital (No. 2015QN026).

\section{CONFLICTS OF INTEREST}

The authors of this article declare no conflicts of interest.

\section{REFERENCES}

1. Munoz JJ, Ellison LM. Upper tract urothelial neoplasms: incidence and survival during the last 2 decades. J Urol. 2000; 164:1523-5.
2. Roupret M, Babjuk M, Comperat E, Zigeuner R, Sylvester RJ, Burger M, Cowan NC, Bohle A, Van Rhijn BW, Kaasinen E, Palou J, Shariat SF. European Association of Urology Guidelines on Upper Urinary Tract Urothelial Cell Carcinoma: 2015 Update. Eur Urol. 2015; 68:868-79. doi: 10.1016/j.eururo.2015.06.044.

3. Straub J, Strittmatter F, Karl A, Stief CG, Tritschler S. Ureterorenoscopic biopsy and urinary cytology according to the 2004 WHO classification underestimate tumor grading in upper urinary tract urothelial carcinoma. Urol Oncol. 2013; 31:1166-70. doi: 10.1016/j.urolonc.2011.12.021.

4. Messer J, Shariat SF, Brien JC, Herman MP, Ng CK, Scherr DS, Scoll B, Uzzo RG, Wille M, Eggener SE, Steinberg G, Terrell JD, Lucas SM, et al. Urinary cytology has a poor performance for predicting invasive or highgrade upper-tract urothelial carcinoma. BJU Int. 2011; 108:701-5. doi: 10.1111/j.1464-410X.2010.09899.x.

5. Skacel M, Fahmy M, Brainard JA, Pettay JD, Biscotti CV, Liou LS, Procop GW, Jones JS, Ulchaker J, Zippe CD, Tubbs RR. Multitarget fluorescence in situ hybridization assay detects transitional cell carcinoma in the majority of patients with bladder cancer and atypical or negative urine cytology. J Urol. 2003; 169:2101-5. doi: 10.1097/01. ju.0000066842.45464.cc.

6. Bubendorf L, Grilli B, Sauter G, Mihatsch MJ, Gasser TC, Dalquen P. Multiprobe FISH for enhanced detection of bladder cancer in voided urine specimens and bladder washings. Am J Clin Pathol. 2001; 116:79-86. doi: 10.1309/ K5P2-4Y8B-7L5A-FAA9.

7. Hara I, Hara S, Miyake H, Nomi M, Gotoh A, Kawabata G, Arakawa S, Kamidono S. Usefulness of Ureteropyeloscopy for diagnosis of upper urinary tract tumors. J Endourol. 2001; 15:601-5. doi: 10.1089/089277901750426373.

8. Keeley FX, Kulp DA, Bibbo M, McCue PA, Bagley DH. Diagnostic accuracy of ureteroscopic biopsy in upper tract transitional cell carcinoma. J Urol. 1997; 157:33-7.

9. Lim DJ, Shattuck MC, Cook WA. Pyelovenous lymphatic migration of transitional cell carcinoma following flexible ureterorenoscopy. J Urol. 1993; 149:109-11.

10. Luo HL, Kang CH, Chen YT, Chuang YC, Lee WC, Cheng YT, Chiang PH. Diagnostic ureteroscopy independently correlates with intravesical recurrence after nephroureterectomy for upper urinary tract urothelial carcinoma. Ann Surg Oncol. 2013; 20:3121-6. doi: 10.1245/s10434-013-3000-z.

11. Sung HH, Jeon HG, Han DH, Jeong BC, Seo SI, Lee HM, Choi HY, Jeon SS. Diagnostic Ureterorenoscopy Is Associated with Increased Intravesical Recurrence following Radical Nephroureterectomy in Upper Tract Urothelial Carcinoma. PLoS One. 2015; 10:e0139976. doi: 10.1371/journal. pone.0139976.

12. Lee JK, Kim KB, Park YH, Oh JJ, Lee S, Jeong CW, Jeong SJ, Hong SK, Byun SS, Lee SE. Correlation Between the Timing of Diagnostic Ureteroscopy and Intravesical Recurrence in Upper Tract Urothelial Cancer. Clinical Genitourinary Cancer. 2016; 14:E37-E41. doi: 10.1016/j. clgc.2015.07.008. 
13. Melamed MR, Reuter VE. Pathology and staging of urothelial tumors of the kidney and ureter. Urol Clin North Am. 1993; 20:333-47.

14. Johannes JR, Nelson E, Bibbo M, Bagley DH. Voided urine fluorescence in situ hybridization testing for upper tract urothelial carcinoma surveillance. J Urol. 2010; 184:879-82. doi: 10.1016/j.juro.2010.05.023.

15. Chen AA, Grasso M. Is there a role for FISH in the management and surveillance of patients with upper tract transitional-cell carcinoma? J Endourol. 2008; 22:1371-4. doi: 10.1089/end.2008.0096.

16. Chen GL, El-Gabry EA, Bagley DH. Surveillance of upper urinary tract transitional cell carcinoma: the role of ureteroscopy, retrograde pyelography, cytology and urinalysis. J Urol. 2000; 164:1901-4.

17. Akkad T, Brunner A, Pallwein L, Gozzi C, Bartsch G, Mikuz G, Steiner H, Verdorfer I. Fluorescence in situ hybridization for detecting upper urinary tract tumors-a preliminary report. Urology. 2007; 70:753-7. doi: 10.1016/j.urology.2007.06.1103.

18. Marin-Aguilera M, Mengual L, Ribal MJ, Musquera M, Ars E, Villavicencio H, Algaba F, Alcaraz A. Utility of fluorescence in situ hybridization as a non-invasive technique in the diagnosis of upper urinary tract urothelial carcinoma. Eur Urol. 2007; 51:409-15; discussion 15. doi: 10.1016/j.eururo.2006.08.045.

19. Lodde M, Mian C, Wiener H, Haitel A, Pycha A, Marberger M. Detection of upper urinary tract transitional cell carcinoma with ImmunoCyt: a preliminary report. Urology. 2001; 58:362-6.

20. Cukuranovic R, Ignjatovic I, Visnjic M, Velickovic LJ, Petrovic B, Potic M, Stefanovic V. Characteristics of upper urothelial carcinoma in an area of Balkan endemic nephropathy in south Serbia. A fifty-year retrospective study. Tumori. 2010; 96:674-9.

21. Fang D, Zhang L, Li X, Xiong G, Chen X, Han W, He Z, Zhou L. Risk factors and treatment outcomes of new contralateral upper urinary urothelial carcinoma after nephroureterectomy: the experiences of a large Chinese center. J Cancer Res Clin Oncol. 2014; 140:477-485.

22. Fang D, Liu P, Li X, Xiong G, Zhang L, Singla N, Zhao G, He Q, He Z, Zhou L. Characteristics and treatment outcomes of pan-urothelial cell carcinoma: a descriptive analysis of 45 patients. Sci Rep. 2015; 5:18014. 